\title{
Geomagnetic Variation due to the Solar Eclipse of June 20th, 1955 ।
}

\author{
By Masaziro OTA and Shoichiro HASHIZUME \\ Aso Magnetic Observatory, Kyoto University
}

(Read Oct. 29,1955, Received Sep. 5, 1956)

\begin{abstract}
In order to investigate the geomagnetic variaton due to the Solar Eclipse of June 20th, 1955, the $S_{q}$-field at $13^{h} 30^{m}$ of this day is expressed by a simple, and the current-system of the additional field due to the eclipse is drawn, using the present data and a quantitative result on the bygone eclipse.
\end{abstract}

\section{Introduction}

Statistical and theoretical treatments on geomagnetic variation during solar eclipses have been attempted, but it can hardly be said that the results from both treatments show good agreement with each other. Most of statistical treatments based on observational data were unsuccessful owing to the characteristic irregularity of the $S_{q}$-field. The theoretical ones dealt with an ideal case and its modification [1], but the actual phenomena are too complex to be explained by an ideal expression. In order to carry out a detailed investigation of the effect of the solar eclipse of June 20th, 1955, a temporary observatory was set up at Naze $\left(129^{\circ} 30^{\prime} 9 \mathrm{E}, 28^{\circ} 22^{\prime} 1 \mathrm{~N}\right.$, Amami-Oshima, one of the South-western Islands in Japan), in addition to the existing observatories. The day was magnetically quiet and very favourable for detecting the eclipse-effect. The results of investigation are reported, on the basis of observed data at the several stations observing this eclipse.

\section{Original Data and the Normal Curve}

As a first step towards detecting the magnetic variation due to the solar eclipse, we must determine the normal curve (the $S_{q}$-variation on the eclipse-day assumed to be free from the eclipse-effect). The normal curve is conventionally determined by the mean curve of several quiet days before and after the eclipse-day. As the $S_{q}$-variation at the middle latitude changes its intensity and its form day by day, this mean curve does not always represent the normal curve. The author attempted to find out the eclipse effect by applying a part of normal curve for a few hours, determined from an analysis of all the available observational data around Japan.

Fig. 1 shows the vector-diagrams of the magnetic variation observed on that day at the following Japanese stations: Memambetsu (M), Kakioka (K), Aso (A) and Naze $(\mathrm{N})$, and their stations are shown in Fig. 2. From Fig. 1 it can be seen that the horizontal vectors of these observatories take almost constant values (about 30 gamma and the direction is $10^{\circ} \sim 20^{\circ}$ north from west) during two hours centred at $13^{\mathrm{h}} 30^{\mathrm{m}}$ 


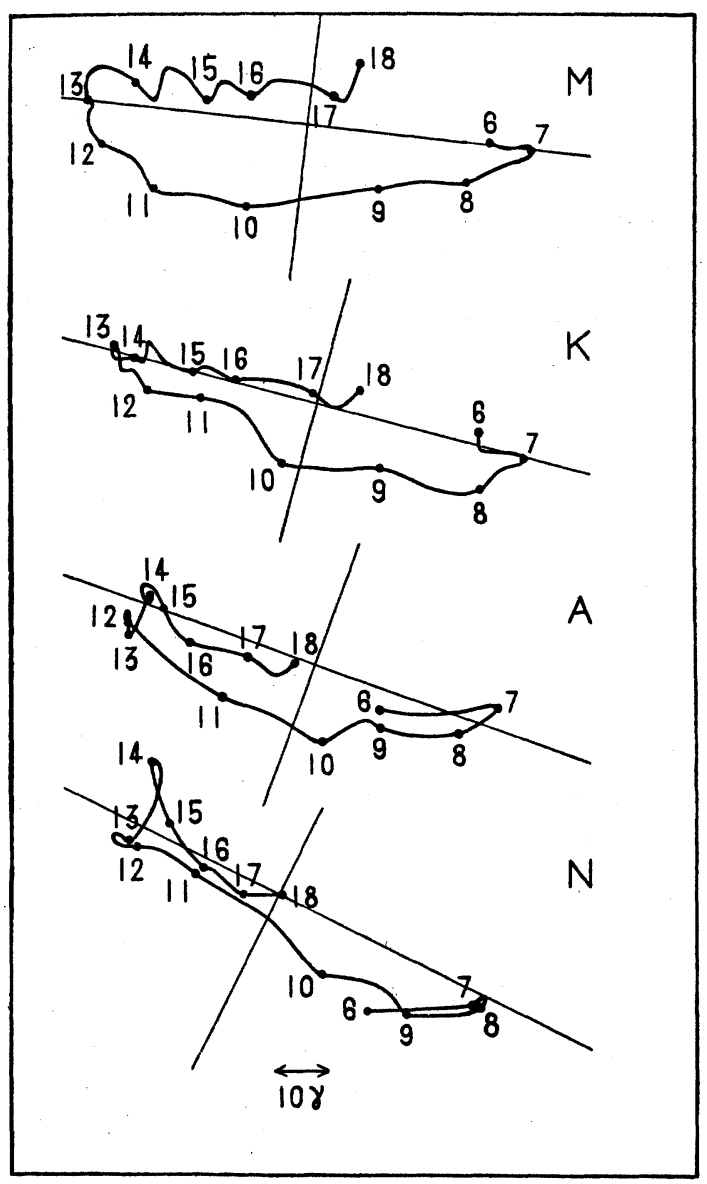

Fig. 1 Vector Diagrams
J.S.T., when the maximum obscuration occurs at this region. This suggests that the general trend of the $S_{q}$-field at this region is approximately uniform during these two hours. This fact is very favourable for the application of a special method for the normal curve. The general trend of the $S_{q}$-field at $13^{\mathrm{h}} 30^{\mathrm{m}}$ J.S.T. is shown in Fig. 2, using the data of the observatories in Japan and Kodaikanal in India. In this case, the normal curve of the station is easily deduced from the curves of the consecutive stations as follows: the currentsystem equivalent to this field is expressed by parallel current and its direction is about $10^{\circ} \sim 20^{\circ}$ east from the Mag. netic North, therefore the normal curves at these five stations can be considered as the same af five stations situated in the northern quarter of the eclipse-area. For the sake of simplicity we introduce a new coordinate-systems as parallel $\left(X^{\prime}\right)$ and perpendicular ( $\left.Y^{\prime}\right)$ to the $S_{q}$. current. The vector-diagram and the

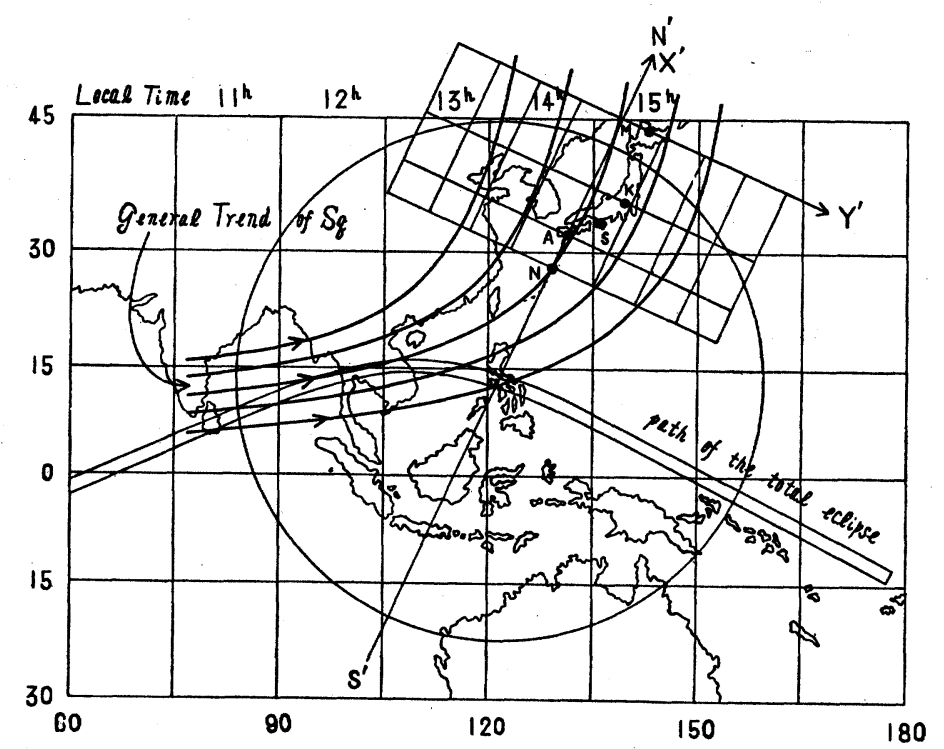

Fig. 2. General State at $13^{h} 30^{m}$ J.S.T. June 20th, 1955. 
curve of Memambetsu seems to undergo so little effect of the eclipse that we may neglect it. Then the normal curves of the other stations are deduced from the Memambetsu-curve. Strictly speaking, the general trend of the $S_{q}$-field is not perfectly parallel and is not in a straight line for the whole region considered here. To determine the normal curve, it is necessary to introduce some corrections due to the difference between local times and the amplitude deduced from variations at the forenoon (right-side of the vector-diagrams) uneffected due to the eclipse. As the $X^{\prime}$-axis is parallel to the general trend to the $S_{q}$-field on this day, the $X^{\prime}$-component of geomagnetic variation on this day could be taken as the eclipse-effect. But this is not satisfactory either, for the above mentioned reason about the general trend of the $S_{q}$-field. From the theoretical consideration as first shown by Chapman [1], we may assume that there are no effects at the hour of the maximum obscuration at each station. Our analyses are based on this assumption.

\section{The Additional Field due to the Eclipse}

The progressing velocity of the eclipse shadow is so slow that the additional field may be considered as a steady state. Therefore the current-system equivalent to this field is to be closed. In order to investigate the current-system of the additional field, it is necessary to have observations sufficiently distributed in the eclipse-area in a large circle as shown in Fig. 2, whose diameter is about 7,000 km. We have only five (as shown in Fig. 2 by black circles), and their distributions are arranged along the $X^{\prime}$. component and not along the $Y^{\prime}$. Fortunately, the $Y^{\prime}$-coordinate is almost parallel to the progressing direction of the eclipse-area on the duration considered here $\left(12^{\mathrm{h}} 30^{\mathrm{m}}\right.$. $14^{\mathrm{h}} 30^{\mathrm{m}}$, or two hours centred at the maximum obscuration in the Japanese district). If we permit an assumption that eclipse effects at all points on the line parallel to the $Y$ '-axis are similar to each other, we can determine magnetic forces corresponding to the eclipse effect at the cross-points of the rectangular mesh by the $X^{\prime}$ - $Y^{\prime}$-coordinatesystem, using the curves of four stations (Shimosato is omitted, as it is situated too close to Aso), and they are shown in Fig. 3A. To secure this figure, we applied the following consideration for each station: variations after the hour of the maximum obscuration represent the eclipse-effect at the rear-side (left, in this figure) of that station, and similary at the opposite side.

The current-system equivalent to the additional field due to the eclipse is derived from the horizontal vectors of this field. This field has a potential, but all these vectors do not satisfy this condition, as they introduce some errors. Then, in practice, the two figures corresponding to this field are drawn; one is derived from $X^{\prime}$-component (3B) and the other from the $Y^{\prime}(3 \mathrm{C})$. As to the disagreement of those two figures, the method adopted by Hasegawa and Ota is introduced [2]. This method signifies the mean condition of the two figures, (3B) and (3C), and the result is shown in Fig. 3D. All Fig. 3's are the additional field due to the eclipse at the northern quarter of the eclipse-area, and the potential-difference of the consecutive line is $2.2 \times 10^{3} \mathrm{c}$.g.s., and the arrows show the direction of the current equivalent to the additional field. Next, the 


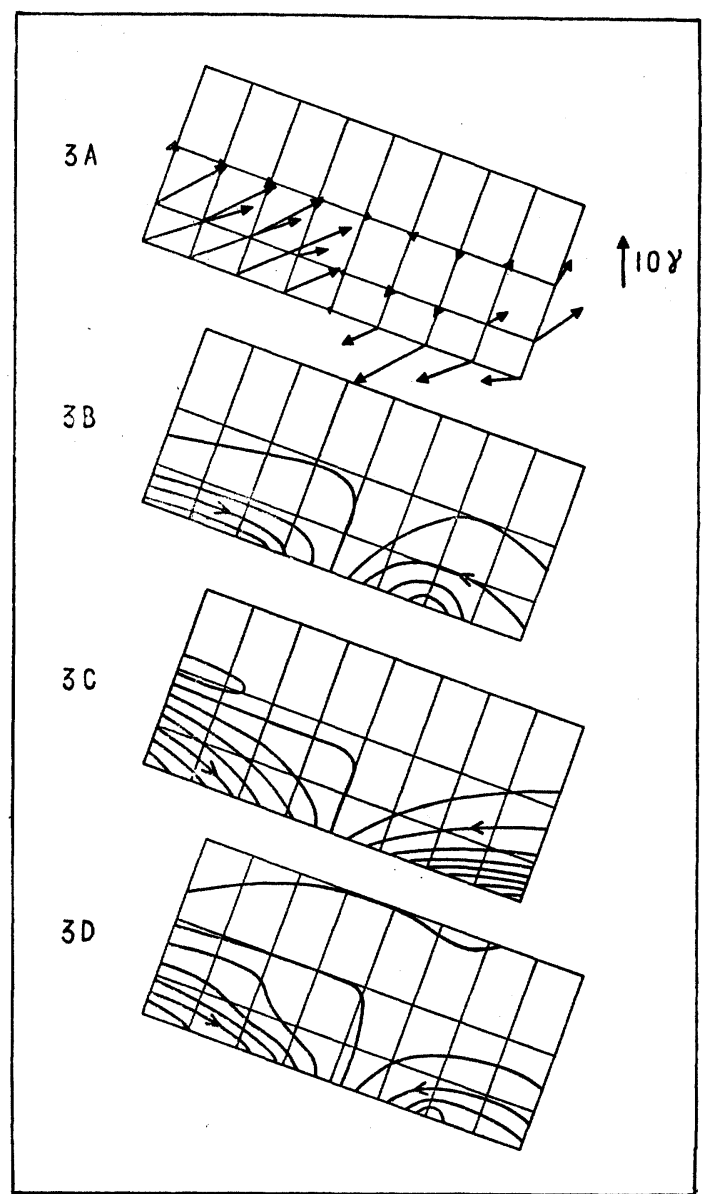

Fig. 3 Northern Quarter of Additional Field due to the eclipse. additional field at the central part of the eclipse-area is considered. The general trend of the $S_{q}$-field at the central part of the present eclipse-area is not so simple as that of the northern quarter, as shown in Fig. 2. Then we utilize the previous result from the total eclipse, in which the diminution of the magnetic force was about half of the normal $S_{q}$ at that hour, and assume that the general trend of the $S_{q}$-field is spread in the same manner at the northern quarter. Fig. 4 shows the half-side of the additional field, obtained by combining the field thus obtained with the field shown in Fig. 3D. This result gives a typical example of the additional field due to ehe eclipse, under the condition of the uniform $S_{q}$-field.

\section{Conclusion}

The eclipse-effect depends on the condition of the variation field, and the affected area exceeds the eclipse-area, as the present result shows. It is difficult to draw the general picture of the
Fig. 4 Northern Half of Additional Field Potential Difference of consecutive lines is $2.2 \times 10^{3}$ c.g.s.

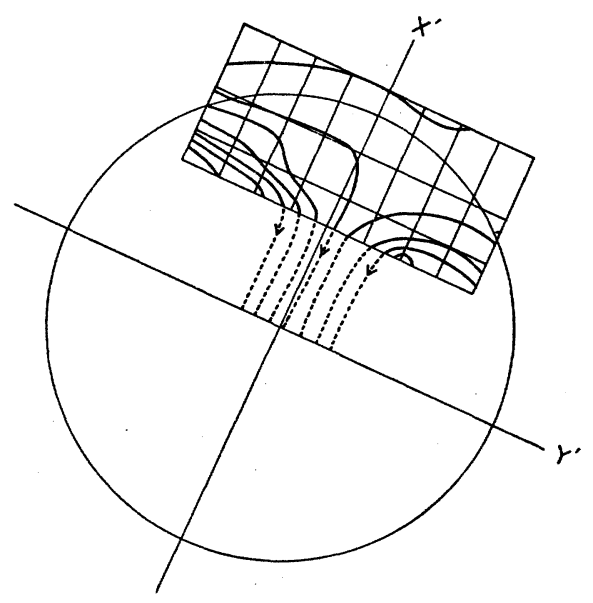


additional field due to the eclipse, as the variation field is too complex to be expressed by a simple model.

In conclusion, the writer wishes to express his sincere thanks to Prof. M. Hasegawa for his encouragement of this work, and also to Dr. A.K. Das, the Director General of Observatories sending us the observational data obtained in India.

\section{References}

[1] S. Chapman; Terr. Mag., 38, 175 (1933).

T. Nagata, Y. Nakata, T. Rikitake and I. Yokoyama; Rep. Ionosphere Res. Japan, 9, 121 (1955). etc.

[2] M. Hasegawa and M. Ota ; I.A.T.M.E. Bull. No. 13, Trans. of Oslo Meeting, 431 (1950). 